\title{
Contrast-Related Neurotoxicity: An Entity Not to be Forgotten
}

\author{
Rengarajan Rajagopal ${ }^{1, \odot}$ Sreenivasa Narayana Raju ${ }^{1} \quad$ Niraj Nirmal Pandey ${ }^{1} \quad$ Sanjiv Sharma ${ }^{1}$ \\ ${ }^{1}$ Department of Cardiovascular Radiology and Endovascular \\ Interventions, Cardiothoracic Neurosciences Center, All India \\ Institute of Medical Sciences, Ansari Nagar, New Delhi, India

\begin{abstract}
Address for correspondence Rengarajan Rajagopal, MD, DNB, Room No. 10A, Department of Cardiovascular Radiology and Endovascular Interventions, Cardiothoracic Neurosciences Center, All India Institute of Medical Sciences, Ansari Nagar, New Delhi 110029, India (e-mail: heraghava@gmail.com).
\end{abstract}
Abstract
Keywords
- iodinated contrast
- neurotoxicity
- angiography

\begin{abstract}
Neurotoxicity related to intra-arterial use of iodinated contrast agents is a relatively uncommon complication of angiography. The authors describe the case of a 42-year-old patient with type B aortic dissection, who developed contrast-related neurological symptoms.
\end{abstract}

\section{Discussion}

A 42-year-old man with no prior cardiovascular comorbidities presented with acute severe chest pain and history of dyspnea for the past 6 months. Routine blood investigations were normal with normal levels of cardiac biomarkers. Preliminary echocardiography performed in the emergency room showed aortic regurgitation with a dissection flap in the visualized descending aorta. Emergency computed tomographic (CT) angiography showed a type B aortic dissection, with pseudoaneurysm formation at the proximal descending thoracic aorta ( - Fig. 1). In view of continuing severe chest pain and false lumen pseudoaneurysm, decision was taken for endovascular stent graft placement to cover the entry tear. In the angiography suite, after the initial aortic angiograms for planning the device placement, the patient suddenly developed drowsiness and responded to commands with incomprehensible words. Doppler evaluation of the carotid arteries was performed and was normal. Without further delay, the patient was intubated and the procedure was completed ( $\mathbf{- F i g s .} \mathbf{2}, \mathbf{3}$ ). The total amount of iodinated contrast (nonionic) used in the procedure was $300 \mathrm{~mL}$. On extubation, the patient was still found to be drowsy with incomprehensible sounds. Noncontrast CT of the head was performed, which showed focal cortical edema and sulcal hyperdensities in the left high frontal lobe and parietal lobe ( - Fig. 4). The patient was closely observed, and his symptoms resolved after 36 hours.

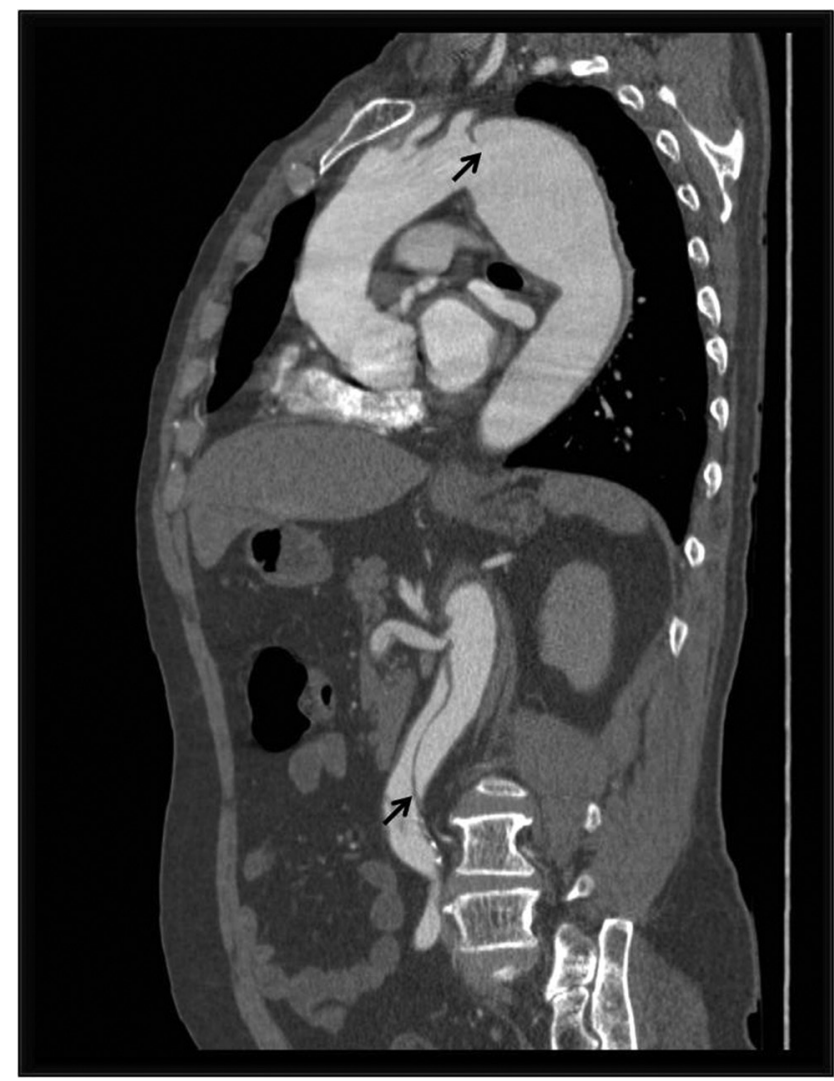

Fig. 1 Sagittal oblique image of the CT angiogram showing the type B dissection with the dissection flap (black arrows) in the descending thoracic and abdominal aorta.

Dr. Rengarajan Rajagopal's ORCID is https://orcid.org/0000-0003-0037-1357

\section{received}

December 30, 2018

accepted after revision

April 8, 2019

published online

July 15, 2019
DOI https://doi.org/

10.1055/s-0039-1693539

ISSN 2457-0214.
(C2019 by Indian Society of Vascular and Interventional Radiology
License terms

() (1) $\Theta \circledast$ 


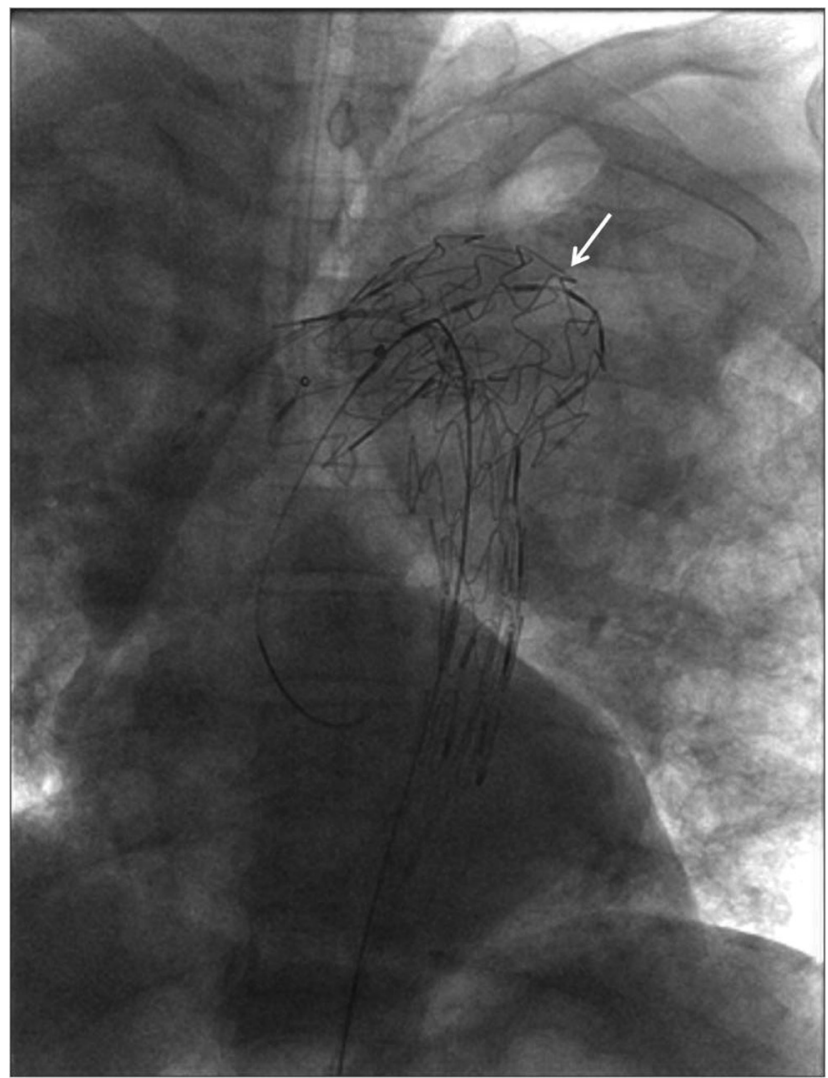

Fig. 2 Fluoroscopic image showing the stent graft (white arrow) deployed to cover the entry tear.

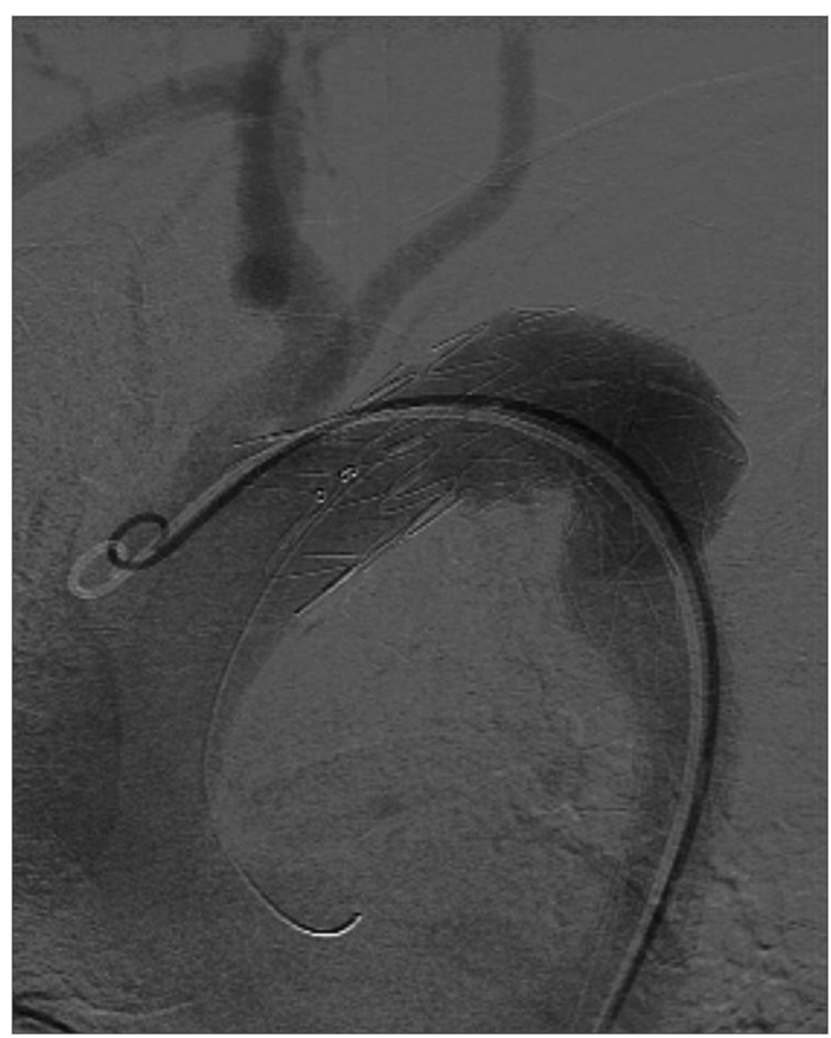

Fig. 3 Angiographic image post stent graft deployment showing absence of filling of the false lumen and no flow in the left subclavian artery (covered by the stent graft).

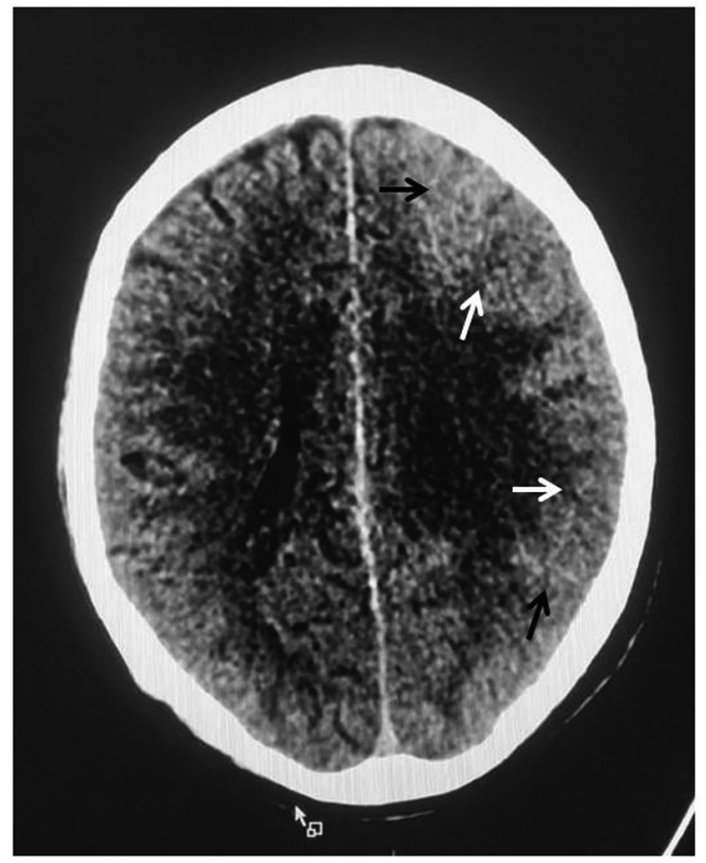

Fig. 4 Axial section of noncontrast $\mathrm{CT}$ of the brain showing focal cortical edema (white arrows) and sulcal hyperdensities (black arrows).

Neurotoxicity related to intra-arterial use of iodinated contrast agents is a relatively uncommon complication of angiography, usually seen after neurointerventional procedures and coronary angiographies. It is thought to result from a transient disruption of the blood-brain barrier after multiple contrast injections into the same vessel. Hypertension and renal failure are important predisposing risk factors, in addition to the total amount and osmolarity of the contrast agent. ${ }^{1}$ Presenting most commonly as transient cortical blindness, encephalopathy and seizures are the other common presenting symptoms. Diagnosis of contrast-related neurotoxicity becomes important, as it needs to be differentiated from embolic and hemorrhagic complications related to the procedure. CT scan of the head, when performed early, shows features such as abnormal cortical contrast enhancement and edema, subarachnoid contrast enhancement, and striatal contrast enhancement., ${ }^{2,3}$ Magnetic resonance imaging may show hyperintense areas on FLAIR (fluid-attenuated inversion recovery) and diffusion-weighted images. Contrast-related neurotoxicity usually follows a benign clinical course usually resolving within 48 hours; however, cases with permanent neurological deficits and fatal cerebral edema have been reported in the literature. ${ }^{4}$

In conclusion, patients who develop neurological symptoms after angiography require close observation and evaluation for a diagnosis of contrast-induced neurotoxicity and to rule out other embolic and hemorrhagic complications. Appropriate management measures for reduction in cerebral edema, when instituted early, help in improvement of symptoms and reduction of morbidity.

\section{Conflicts of Interest}

None declared. 


\section{References}

1 Leong S, Fanning NF. Persistent neurological deficit from iodinated contrast encephalopathy following intracranial aneurysm coiling. A case report and review of the literature. Interv Neuroradiol 2012;18(1):33-41

2 Baik SK, Kim YS, Lee HJ, Park J, Kim GC. Immediate CT findings following embolization of cerebral aneurysms: suggestion of blood-brain barrier or vascular permeability change. Neuroradiology 2008;50(3):259-266
3 Brisman JL, Jilani M, McKinney JS. Contrast enhancement hyperdensity after endovascular coiling of intracranial aneurysms. AJNR Am J Neuroradiol 2008;29(3):588-593

4 Gollol Raju NS, Joshi D, Daggubati R, Movahed A. Contrast induced neurotoxicity following coronary angiogram with Iohexol in an end stage renal disease patient. World J Clin Cases 2015;3(11):942-945 